\title{
Avaliação pós-operatória de pacientes submetidos a reparo artroscópico de instabilidade anterior do ombro
}

\section{Postoperative Evaluation of Patients Submitted to Arthroscopic Repair of Anterior Shoulder Instability}

\author{
Thiago Medeiros Storti ${ }^{1,2}$ Renato de Almeida Lima ${ }^{1}$ Eloíse de Barros e Silva Costa ${ }^{2,3}$

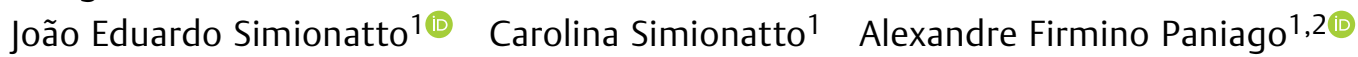 \\ 1 Instituto do Ombro de Brasília, Brasília, DF, Brasil \\ ${ }^{2}$ Grupo do Ombro, Instituto de Pesquisa e Ensino HOME (IPE HOME), \\ Brasília, DF, Brasil \\ 3 Departamento de Ortopedia, Centro Universitário de Brasília \\ (UniCEUB), Brasília, DF, Brasil \\ Endereço para correspondência Thiago Medeiros Storti, MD, Quadra \\ 102 norte, praça Perdiz, Lote 05, Condomínio Residencial Matisse \\ Antares, Águas Claras, DF, 71.907-000, Brasil \\ (e-mail: thiago_storti@hotmail.com).
}

Rev Bras Ortop 2020;55(3):339-346.

\section{Resumo \\ Palavras-chave \\ - artroscopia \\ - instabilidade articular \\ - ombro \\ - estudos retrospectivos}

Objetivo Avaliar clinicamente os resultados de pacientes submetidos a tratamento cirúrgico artroscópico de instabilidade anterior do ombro.

Métodos Estudo retrospectivo de 94 pacientes. Com seguimento mínimo de 24 meses, buscamos correlacionar as características dos pacientes e da cirurgia, como idade, gênero, tipo de lesão (traumática ou atraumática) e posição do paciente na cirurgia (decúbito lateral e cadeira de praia) com os resultados obtidos, avaliando o índice de recidivas de luxação, a perda de rotação lateral, a dor residual, e os escores funcionais de Carter-Rowe, da University of California at Los Angeles (UCLA) e de Constant-Murley.

Resultados Observamos uma taxa de recidiva de luxação de $11,7 \%$, perda de rotação lateral em $37,23 \%$ dos pacientes, e algum grau de dor residual em $51,6 \%$. Obtivemos uma pontuação média no escore de Carter Rowe de 85,37 , representando $86 \%$ de resultados bons/excelentes. No escore da UCLA, obtivemos $88 \%$ de resultados bons/excelentes, índice semelhante aos encontrados no escore de Constant-Murley (86\%).

Conclusão $\mathrm{O}$ tratamento artroscópico da instabilidade anterior do ombro apresenta resultados satisfatórios e baixo índice de complicações importantes, podendo ser o método de escolha para a maioria dos pacientes.

Objective To clinically evaluate the results of patients undergoing arthroscopic surgical treatment of anterior shoulder instability.

Methods A retrospective study of 94 patients. With a minimum follow-up of 24 months, we sought to correlate the characteristics of the patients and the surgery, such as age, gender, type of injury (traumatic or atraumatic) and the patient's position during surgery (lateral decubitus and beach chair) with the results obtained, the recurrence rate, the lateral recebido

04 de Abril de 2018

aceito

05 de Fevereiro de 2019
DOI https://doi.org/

10.1055/s-0039-3402467. ISSN 0102-3616.
Copyright $\odot 2020$ by Sociedade Brasileira License terms de Ortopedia e Traumatologia. Published by Thieme Revinter Publicações Ltda, Rio de Janeiro, Brazil 
rotation loss, the residual pain, and the functional scores of Carter-Rowe, University of California at Los Angeles (UCLA), and Constant-Murley.

Results We observed a recurrent dislocation rate of $11.7 \%$, lateral rotation loss in $37.23 \%$ of the patients, and some degree of residual pain in $51.6 \%$ of them. We obtained a mean Carter Rowe score of 85.37 , representing $86 \%$ of good/excellent results. In the UCLA score, we obtained $88 \%$ of good/excellent results, similar to those obtained in the Constant-Murley score (86\%).

Conclusion The arthroscopic treatment of the anterior instability of the shoulder presents satisfactory results and low index of important complications, being the method of choice for most patients.

\section{Introdução}

O tipo mais comum de luxação glenoumeral é a anterior, determinada pela lesão do complexo capsulolabral na porção anteroinferior da glenoide, conhecida como lesão de Bankart, e também chamada de lesão essencial. ${ }^{1-4}$

Nos casos de luxações recorrentes, é indicado o reparo cirúrgico, podendo este ser realizado de forma aberta ou artroscópica. Entre os tratamentos cirúrgicos existentes para estabilizar o ombro, o reparo por via artroscópica tem se tornado cada vez mais popular devido a vantagens como menor perda de movimento, menor agressão cirúrgica, preservação do subescapular e baixa morbidade, em comparação com a cirurgia aberta. ${ }^{5}$

Vários fatores relacionados ao paciente que contribuem para o risco de recorrência após o reparo artroscópico de Bankart têm sido relatados, incluindo idade mais jovem no momento da cirurgia, gênero masculino, instabilidade bilateral, hiperfrouxidão ligamentar, participação em esportes de colisão, e o retorno precoce para esportes de contato. ${ }^{6}$ Fatores de risco associados à lesão, como erosão ou deficiência da glenoide, o tamanho da lesão de Hill-Sachs, e se a lesão está envolvendo a borda anterior da glenoide também foram implicados. ${ }^{7}$ A identificação precisa dos fatores de risco associados ao insucesso do reparo artroscópico de Bankart e do deslocamento capsular oferece ao cirurgião a capacidade de estratificar o risco para o paciente individualmente, e permite o aconselhamento adequado.

Deste modo, o objetivo do presente estudo é a avaliação funcional e a análise dos fatores que influenciam nos resultados de pacientes submetidos ao tratamento artroscópico da instabilidade anterior do ombro.

\section{Materiais e Métodos}

No período de março a maio de 2017, foram analisados retrospectivamente 94 pacientes por meio de revisão de prontuários e posterior avaliação clínica. Todos os pacientes foram submetidos ao tratamento cirúrgico artroscópico da instabilidade anterior do ombro em dois hospitais particulares e por quatro cirurgiões independentes, entre janeiro de 2010 e dezembro de 2014 . No total, dez pacientes operados neste período foram excluídos pelo não comparecimento para revisão.
Os prontuários apresentavam as seguintes informações: identificação do paciente, anamnese caracterizando a causa da instabilidade (traumática ou atraumática), limitação funcional, exame físico pré-operatório, exames de imagem préoperatórios, e descrição cirúrgica. A avaliação pós-operatória teve um seguimento de 24 a 72 meses, com média de 40 meses, buscando verificar pela clínica e pelo exame físico a limitação funcional, a diminuição da rotação lateral, a instabilidade e dor, além da aplicação dos escores funcionais de Carter-Rowe, da University of California at Los Angeles (UCLA) e de ConstantMurley aos pacientes por um examinador externo.

Foram incluídos neste estudo pacientes que apresentavam instabilidade anterior recorrente (luxação ou subluxação) e lesão de Bankart, todos operados por via artroscópica e com um mínimo de 24 meses de acompanhamento pós-operatório. Entre os critérios de exclusão utilizados, se encontram os casos de luxação traumática associada a lesão neurovascular, fratura acometendo outros locais da cintura escapular, perda óssea maior do que $25 \%$ da glenoide, fratura de Hill-Sachs envolvendo mais de $1 / 4$ da cabeça do úmero, cirurgias prévias no ombro envolvido, e instabilidade multidirecional.

No total, dois cirurgiões realizaram as cirurgias com o paciente em decúbito lateral. O procedimento cirúrgico é realizado sob anestesia geral e bloqueio de plexo braquial. $\mathrm{O}$ membro é mantido em abdução de aproximadamente $70^{\circ} \mathrm{e}$ flexão de $20^{\circ}$, aplicando-se tração longitudinal fixa e vertical com pesos entre $4 \mathrm{~kg}$ e7 $\mathrm{kg}$.

Os outros dois cirurgiões realizaram as cirurgias com o paciente em posição de cadeira de praia, na qual ele fica com elevação do tronco de pelo menos $70^{\circ}$, com flexão de joelho em torno de $30^{\circ}$, e com inclinação lateral suave oposta ao lado operado.

Os portais artroscópicos utilizados pelos cirurgiões foram os convencionais (posterior, anterossuperior e anteroinferior). Durante o procedimento, era realizada inspeção articular para avaliar a presença de lesões associadas. Subsequentemente, observava-se a lesão labral anteroinferior e sua extensão, realizava-se liberação capsulolabral e escarificação da borda da glenoide, e, posteriormente, fixava-se a lesão labral utilizando-se de duas a quatro âncoras bioabsorvíveis.

No pós-operatório, os pacientes permaneceram com imobilização contínua em tipoia tipo Velpeau pelo período de três semanas. Foram, então, iniciados movimentos pendulares e autopassivos nas duas semanas seguintes, e movimentos de 
rotação lateral além de $20^{\circ}$ a partir da sexta semana. 0 fortalecimento muscular dependeu da amplitude de movimento do ombro operado, e geralmente começava no terceiro mês de pós-operatório. $\mathrm{O}$ retorno às atividades esportivas de contato ou colisão foi liberado a partir do sexto mês.

$\mathrm{Na}$ análise descritiva, os dados observados, expressos pela mediana e pelo intervalo interquartílico (IIQ) para dados contínuos (quantitativos) e pela frequência (n) e pelo percentual (\%) para dados categóricos (qualitativos), foram apresentados em tabelas.

A análise inferencial foi composta pelos seguintes métodos:

- a associação entre os escores Carter-Rowe, da UCLA e de Constant-Murley com as variáveis clínicas (idade, gênero, tipo de lesão e posição da cirurgia) foi avaliada pelo teste de Mann-Whitney para dados categóricos e pelo coeficiente de correlação de Spearman para dados numéricos; e

- a associação entre recidiva da luxação, dor residual, perda da rotação lateral e apreensão com as variáveis clínicas foi analisada pelo teste do qui-quadrado $\left(X^{2}\right)$ ou exato de Fisher para dados categóricos, e pelo teste de MannWhitney para dados numéricos.

Foi aplicado método não paramétrico, pois as variáveis em estudo não apresentaram distribuição normal (Gaussiana), devido à rejeição da hipótese de normalidade pelo teste de Shapiro-Wilks. O critério de determinação de significância adotado foi o nível de $5 \%$. A análise estatística foi processada pelo software estatístico Statistical Analysis System (SAS, SAS Institute, Inc., Cary, North Carolina, EUA), versão 6.11.

Todos os pacientes assinaram o termo de consentimento livre esclarecido. Este estudo foi submetido à avaliação e à aprovação do Comitê de Ética em Pesquisa com Seres Humanos, com número de parecer 2.197.472, CAAE 70807917.6.0000.0023.

\section{Resultados}

Com um seguimento de 2 a 6 anos após a cirurgia, 94 pacientes foram avaliados neste estudo; desses, 74 (78,7\%) eram do gênero masculino, e 20 (21,3\%), do gênero feminino, com a idade no procedimento cirúrgico variando de 17 a 62 anos (-Tabela 1). Entre os pacientes, $11(11,7 \%)$ apresentaram recidiva da luxação, 35 (37,23\%) apresentaram diminuição da rotação lateral, $48(51,06 \%)$ relataram algum grau de dor residual, e $23(24,46 \%)$ apresentaram teste de apreensão positivo (-Tabela 2 ).

A instabilidade traumática foi prevalente, representando $90,4 \%$ dos casos (85 pacientes). O posicionamento cirúrgico mais utilizado foi o de decúbito lateral, sendo 71 dos pacientes operados dessa maneira $(75,5 \%)$ dos pacientes operados dessa maneira. Na avaliação clínica usando o escore de Carter-Rowe, obtivemos a pontuação mediana de 95 (- Tabela 3), sendo 67 (71,3\%) resultados excelentes, $14(14,9 \%)$ bons, $2(2,1 \%)$ regulares e $11(11,7 \%$ ) ruins (-Figura 1). Os resultados ruins foram associados à recidiva da luxação, ocorrendo em 9 pacientes do gênero masculino (81,8\%) e em 2 pacientes do gênero feminino (18,2\%). Apesar de ser mais prevalente em pacientes do gênero masculino, em nosso estudo não foi encontrada diferença
Tabela 1 Descrição geral das variáveis

\begin{tabular}{|c|c|c|}
\hline Variável & $\mathbf{N}$ & $\%$ \\
\hline \multicolumn{3}{|l|}{ Gênero } \\
\hline Masculino & 74 & 78,7 \\
\hline Feminino & 20 & 21,3 \\
\hline \multicolumn{3}{|c|}{ Idade na cirurgia (anos) } \\
\hline Mediana (Q1-Q3) & \multicolumn{2}{|c|}{$34(28-43)$} \\
\hline \multicolumn{3}{|l|}{ Tipo de lesão } \\
\hline Traumática & 85 & 90,4 \\
\hline Atraumática & 9 & 9,6 \\
\hline \multicolumn{3}{|l|}{ Posição } \\
\hline Cadeira de praia & 23 & 24,5 \\
\hline Decúbito lateral & 71 & 75,5 \\
\hline \multicolumn{3}{|c|}{ Escore de Carter-Rowe (pontos) } \\
\hline Mediana (Q1-Q3) & \multicolumn{2}{|c|}{$95(80-100)$} \\
\hline \multicolumn{3}{|c|}{$\begin{array}{l}\text { Escore da University of California at Los Angeles (UCLA) } \\
\text { (pontos) }\end{array}$} \\
\hline Mediana (Q1 - Q3) & \multicolumn{2}{|c|}{$33(31-35)$} \\
\hline \multicolumn{3}{|c|}{ Escore Constant (pontos) } \\
\hline Mediana (Q1-Q3) & \multicolumn{2}{|c|}{$95(87-100)$} \\
\hline
\end{tabular}

estatisticamente significativa entre a variável gênero e a recidiva da luxação. Igualmente, não houve correlação significativa entre o escore de Carter-Rowe e a idade na época da cirurgia $\left(r_{s}=0,162 ; p=0,011\right)$.

Na avaliação do escore da UCLA obtivemos 83 pacientes $(88,3 \%)$ com resultados bons/excelentes e 11 pacientes $(11,7 \%)$ com resultados ruins/razoáveis, sendo estes últimos pacientes os mesmos que apresentaram recidivas (- Figura 2). Constatou-se que há uma correlação direta significativa entre o escore da UCLA e a idade do paciente no momento da cirurgia $\left(r_{s}=0,250 ; p=0,015\right)$. Sendo assim, quanto maior a idade, maior a pontuação.

Tabela 2 Descrição geral das variáveis

\begin{tabular}{|l|l|l|}
\hline Reluxação & N & $\%$ \\
\hline Sim & 11 & 11,7 \\
\hline Não & 83 & 88,3 \\
\hline Dor residual & 48 & 51,1 \\
\hline Sim & 46 & 48,9 \\
\hline Não & \multicolumn{2}{|l}{} \\
\hline Perda da rotação lateral & 37,2 \\
\hline Sim & 35 & 62,8 \\
\hline Não & 59 & \\
\hline Apreensão & 23 & 24,5 \\
\hline Sim & 71 & 75,5 \\
\hline Não &
\end{tabular}


342 Avaliação pós-operatória de pacientes submetidos o reparo artroscópico Storti et al.

Tabela 3 Escores funcionais

\begin{tabular}{|c|c|c|c|c|c|c|c|}
\hline \multirow{2}{*}{$\begin{array}{l}\text { Variável } \\
\text { Idade na cirurgia (anos) }\end{array}$} & \multirow{2}{*}{$\begin{array}{l}\mathrm{N} \\
94\end{array}$} & \multirow{2}{*}{$\begin{array}{l}\text { Mediana } \\
34\end{array}$} & \multicolumn{3}{|c|}{ Intervalo interquartílico } & \multirow{2}{*}{$\begin{array}{l}\text { Mínimo } \\
17\end{array}$} & \multirow{2}{*}{$\begin{array}{l}\text { Máximo } \\
62\end{array}$} \\
\hline & & & 27,8 & - & 43 & & \\
\hline \multicolumn{8}{|l|}{ Escore (pontos) } \\
\hline Carter-Rowe & 94 & 95 & 80 & - & 100 & 15 & 100 \\
\hline University of California at Los Angeles (UCLA) & 94 & 33 & 31 & - & 35 & 23 & 35 \\
\hline Constant-Murley & 94 & 95 & 87 & - & 100 & 68 & 100 \\
\hline
\end{tabular}

Na avaliação do escore Constant-Murley, obtivemos 65 pacientes $(69,2 \%)$ com excelentes resultados, 18 bons (19,1\%), 10 razoáveis $(10,7 \%)$ e 1 resultado ruim (1\%) (-Figura 3). Observou-se que não existe correlação estatisticamente significativa dos três escores com o gênero, o tipo de lesão, e a posição da cirurgia.

Além disso, também não foi observada correlação estatisticamente significativa entre as variáveis clínicas (gênero, idade, tipo de lesão e posição da cirurgia) com a recidiva de luxação, a dor residual, a perda da rotação lateral e a apreensão (-Tabelas 4-7). Apenas observou-se que o grupo com dor residual apresentou idade significativamente menor do que o subgrupo sem dor residual $(p=0,016)$.

\section{Discussão}

A escolha adequada do tratamento cirúrgico da instabilidade anterior do ombro deve ser influenciada diretamente por fatores como a idade, luxações pregressas, ocupação, nível de atividade física, frouxidão ligamentar, saúde geral do paciente, grau de acometimento ósseo da cabeça umeral e da glenoide, além da presença de patologias associadas, como lesões do lábio superior (superior labral tear from anterior to posterior, SLAP) e ruptura de tendões do manguito rotador. ${ }^{8}$ A escolha do tratamento mais adequado para cada paciente pode influenciar os resultados, minimizando os riscos de complicações.

Ainda existem controvérsias quanto ao melhor método de reparo das lesões labrais. ${ }^{9}$ Entre os procedimentos anatômicos, destacam-se as cirurgias aberta e artroscópica de Bankart, que visam o restabelecimento da anatomia original do ombro e envolvem o reparo da lesão labral. ${ }^{10}$ A cirurgia aberta de Bankart, realizada com mobilização e fixação do lábrum, ainda é considerada o padrão-ouro para o tratamento da instabilidade anterior. ${ }^{11}$ Entretanto, condições como o tamanho da incisão, risco de fraqueza do músculo subescapular, perda de amplitude de movimento do ombro (principalmente rotação lateral) e dificuldade de acessar outras lesões intra-articulares têm favorecido a escolha do procedimento artróscopico. Outras vantagens da artroscopia são a menor perda sanguínea, menor duração da cirurgia e a maior satisfação estética. ${ }^{12}$

As taxas de recidivas de luxação após a cirurgia artroscópica vêm diminuindo devido aos avanços tecnológicos e o

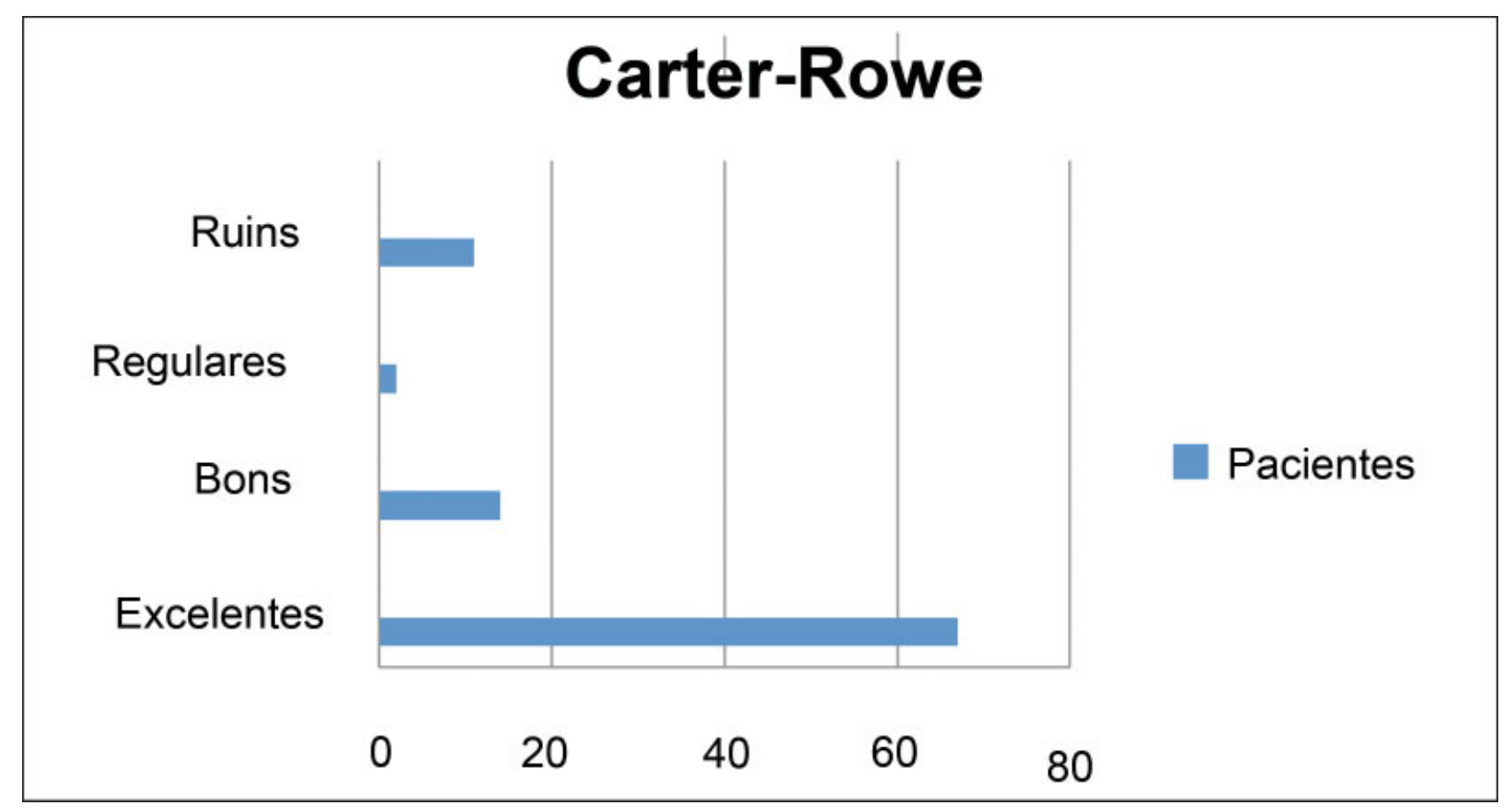

Fig. 1 Escore de Carter-Rowe. 


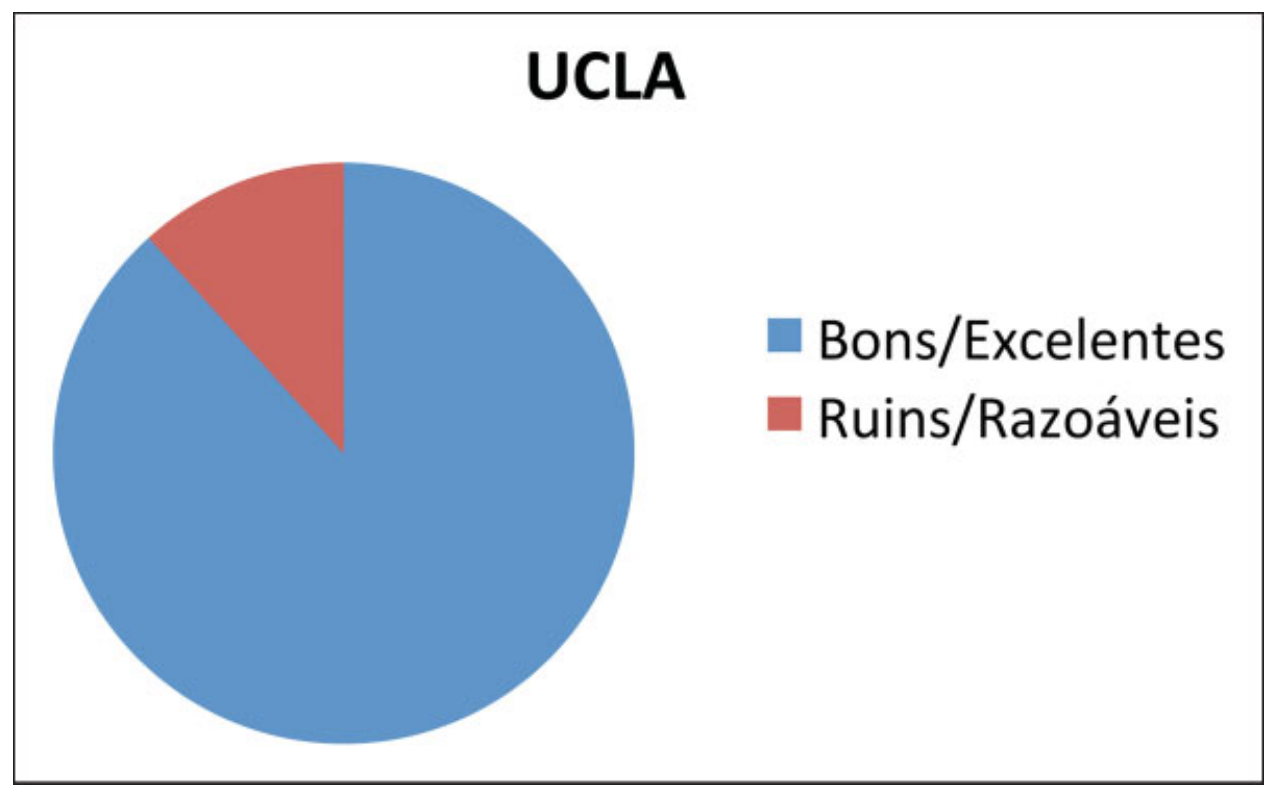

Fig. 2 Escore da University of California at Los Angeles (UCLA).

melhor entendimento dos erros até então cometidos. No ano de 2007, Balg e Boileau ${ }^{13}$ descreveram uma taxa de $14,5 \%$ de recidiva após cirurgia artróscopica de Bankart. Castagna et $\mathrm{al}^{14}$ obtiveram uma taxa de $23 \%$ de recidivas em um seguimento de 10 anos, acompanhando 43 pacientes operados por via artróscopica. Em uma meta-análise, Petrera et al, ${ }^{15}$ ao compararem a cirurgia artroscópica com a aberta de Bankart, encontraram uma taxa de recidiva de luxação $6,3 \%$ menor no grupo artroscópico.

Ahmed et al ${ }^{6}$ também relataram melhores resultados com técnicas artroscópicas, sendo a taxa de recidiva de luxação de $13,2 \%$ em um total de 302 pacientes. Em nosso estudo, todos os casos de recidivas estavam associados à instabilidade traumática, com taxa de $11,7 \%$.
Quanto à perda de rotação lateral, Bottoni et $\mathrm{al}^{16}$ demonstraram uma diminuição de $2^{\circ}$ a $3^{\circ}$ no grupo artroscópico, enquanto, no grupo de cirurgias abertas, houve uma perda de aproximadamente $6,5^{\circ}$ da amplitude de movimento. Em nosso estudo, a perda de rotação lateral foi mais observada nos pacientes que apresentavam instabilidade traumática, dado semelhante aos resultados de Ferreira Neto et al, ${ }^{17}$ que também encontraram maior limitação da rotação lateral em pacientes com essa característica. Tais resultados podem ser justificados pela melhor acomodação capsuloligamentar em pacientes com lesões atraumáticas quando comparados aos pacientes com instabilidade traumática. ${ }^{17-19} \mathrm{Em}$ seu estudo, Almeida Filho et al, ${ }^{20}$ após avaliação de 49 pacientes com análise clínica e radiográfica pós-operatória, observaram que,

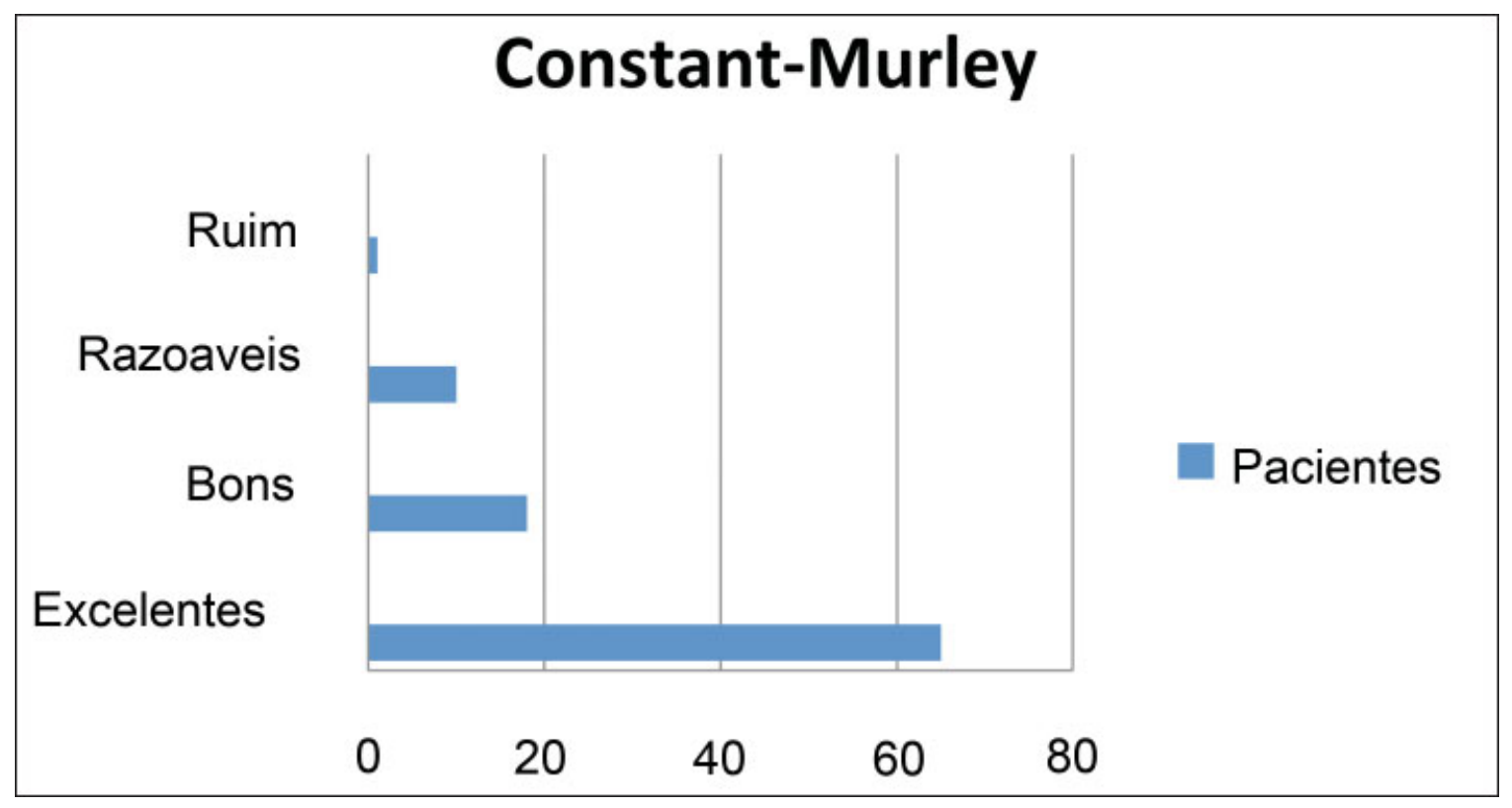

Fig. 3 Escore de Constant-Murley. 
344 Avaliação pós-operatória de pacientes submetidos o reparo artroscópico Storti et al.

Tabela 4 Associação entre as variáveis clínicas e reluxação

\begin{tabular}{|c|c|c|c|c|c|}
\hline Variável & \multicolumn{2}{|c|}{ Com reluxação } & \multicolumn{2}{|c|}{ Sem reluxação } & Valor de $p$ \\
\hline \multicolumn{6}{|l|}{ Gênero } \\
\hline Masculino & 9 & 81,8 & 65 & 78,3 & \multirow[t]{2}{*}{0,57} \\
\hline Feminino & 2 & 18,2 & 18 & 21,7 & \\
\hline \multicolumn{6}{|c|}{ Idade na cirurgia (anos) } \\
\hline Mediana (Q1-Q3) & \multicolumn{2}{|c|}{$32(23-38)$} & \multicolumn{2}{|c|}{$34(28-43)$} & 0,10 \\
\hline \multicolumn{6}{|l|}{ Tipo de lesão } \\
\hline Traumática & 11 & 100 & 74 & 89,2 & \multirow[t]{2}{*}{0,31} \\
\hline Atraumática & 0 & 0 & 9 & 10,8 & \\
\hline \multicolumn{6}{|l|}{ Posição da cirurgia } \\
\hline Cadeira de praia & 4 & 36,4 & 19 & 22,9 & \multirow[t]{2}{*}{0,26} \\
\hline Decúbito lateral & 7 & 63,6 & 64 & 77,1 & \\
\hline
\end{tabular}

Nota: Os dados categóricos foram expressos pela frequência e percentual, e comparados pelo teste do qui-quadrado ou exato de Fisher, e a idade foi expressa pela mediana e intervalo interquartílico, e comparada pelo teste de Mann-Whitney.

Tabela 5 Associação entre as variáveis clínicas e dor residual

\begin{tabular}{|c|c|c|c|c|c|}
\hline Variável & \multicolumn{2}{|c|}{ Com dor residual } & \multicolumn{2}{|c|}{ Sem dor residual } & Valor de $p$ \\
\hline \multicolumn{6}{|l|}{ Gênero } \\
\hline Masculino & 41 & 85,4 & 33 & 71,7 & \multirow[t]{2}{*}{0,085} \\
\hline Feminino & 7 & 14,6 & 13 & 28,3 & \\
\hline \multicolumn{6}{|c|}{ Idade na cirurgia (anos) } \\
\hline Mediana (Q1-Q3) & \multicolumn{2}{|c|}{$32(25-38)$} & \multicolumn{2}{|c|}{$37(30-44)$} & 0,016 \\
\hline \multicolumn{6}{|l|}{ Tipo de lesão } \\
\hline Traumática & 43 & 89,6 & 42 & 91,3 & \multirow[t]{2}{*}{0,52} \\
\hline Atraumática & 5 & 10,4 & 4 & 8,7 & \\
\hline \multicolumn{6}{|l|}{ Posição da cirurgia } \\
\hline Cadeira de praia & 12 & 25,0 & 11 & 23,9 & \multirow[t]{2}{*}{0,9} \\
\hline Decúbito lateral & 36 & 75,0 & 35 & 76,1 & \\
\hline
\end{tabular}

Tabela 6 Associação entre as variáveis clínicas e perda da rotação lateral

\begin{tabular}{|c|c|c|c|c|c|}
\hline Variável & \multicolumn{2}{|c|}{$\begin{array}{l}\text { Com perda da rotação } \\
\text { lateral }\end{array}$} & \multicolumn{2}{|c|}{$\begin{array}{l}\text { Sem perda da rotação } \\
\text { lateral }\end{array}$} & Valor de $p$ \\
\hline \multicolumn{6}{|l|}{ Gênero } \\
\hline Masculino & 29 & 82,9 & 45 & 76,3 & \multirow[t]{2}{*}{0,45} \\
\hline Feminino & 6 & 17,1 & 14 & 23,7 & \\
\hline \multicolumn{6}{|c|}{ Idade na cirurgia (anos) } \\
\hline Mediana (Q1-Q3) & \multicolumn{2}{|l|}{$35(25-44)$} & \multicolumn{2}{|c|}{$34(28-40)$} & 0,53 \\
\hline \multicolumn{6}{|l|}{ Tipo de lesão } \\
\hline Traumática & 30 & 85,7 & 55 & 93,2 & \multirow[t]{2}{*}{0,20} \\
\hline Atraumática & 5 & 14,3 & 4 & 6,8 & \\
\hline \multicolumn{6}{|l|}{ Posição da cirurgia } \\
\hline Cadeira de praia & 6 & 17,1 & 17 & 28,8 & \multirow[t]{2}{*}{0,20} \\
\hline Decúbito lateral & 29 & 82,9 & 42 & 71,2 & \\
\hline
\end{tabular}


Tabela 7 Associação entre as variáveis clínicas e a apreensão

\begin{tabular}{|c|c|c|c|c|c|}
\hline Variável & \multicolumn{2}{|c|}{ Com apreensão } & \multicolumn{2}{|c|}{ Sem apreensão } & Valor de $p$ \\
\hline \multicolumn{6}{|l|}{ Gênero } \\
\hline Masculino & 19 & 82,6 & 55 & 77,5 & \multirow[t]{2}{*}{0,42} \\
\hline Feminino & 4 & 17,4 & 16 & 22,5 & \\
\hline \multicolumn{6}{|c|}{ Idade na cirurgia (anos) } \\
\hline Mediana (Q1-Q3) & \multicolumn{2}{|c|}{$31(25-40)$} & \multicolumn{2}{|c|}{$34(28-43)$} & 0,29 \\
\hline \multicolumn{6}{|l|}{ Tipo de lesão } \\
\hline Traumática & 23 & 100 & 62 & 87,3 & \multirow[t]{2}{*}{0,070} \\
\hline Atraumática & 0 & 0 & 9 & 12,7 & \\
\hline \multicolumn{6}{|l|}{ Posição da cirurgia } \\
\hline Cadeira de praia & 5 & 21,7 & 18 & 25,4 & \multirow[t]{2}{*}{0,72} \\
\hline Decúbito lateral & 18 & 78,3 & 53 & 74,6 & \\
\hline
\end{tabular}

apesar de a diminuição da rotação lateral estar relacionada com a presença de artrose, não foi possível concluir a relação causa-efeito entre ambas.

A dor residual é uma queixa comum encontrada no pósoperatório dos pacientes tratados cirurgicamente para instabilidade anterior do ombro. Vários autores ${ }^{21-23}$ atribuem essa complicação a lesões que estão associadas à instabilidade e podem não ser diagnosticadas em um primeiro momento, sendo essas principalmente rupturas parciais do manguito rotador, lesões SLAP e alterações degenerativas articulares. No nosso estudo, aproximadamente $51 \%$ dos pacientes apresentaram algum grau de dor residual no pós-operatório, sendo observada uma associação inversa entre os fatores idade e dor. Nesse sentido, constatou-se que, quanto menor a idade no momento da cirurgia, maior a ocorrência de dor pós-operatória, o que pode ser justificado pelo maior nível de atividade executado por esse grupo de pacientes. Além disso, conforme observado por Almeida Filho et al, ${ }^{20}$ há relação significativa entre o desenvolvimento de artrose glenoumeral pós-operatória e pacientes mais jovens no momento da cirurgia e da primeira luxação, corroborando nossa observação.

Alguns estudos ${ }^{13,24-27}$ mostraram que pacientes mais jovens apresentam um risco aumentado de recorrência após a estabilização cirúrgica artroscópica, mas nenhuma idade foi definida. Segundo Ahmed et al, ${ }^{6}$ a idade, por si só, não é uma base suficiente para decisões sobre qual tratamento oferecer aos pacientes mais jovens; no entanto, é claramente um fator importante na previsão do risco de recorrência. Em nossa casuística, não houve correlação estatisticamente significativa entre idade e recidiva da luxação, ficando a idade mediana dos pacientes vítimas de recidiva semelhante à dos pacientes sem recidiva (32 anos versus 34 anos).

Nós utilizamos três escores funcionais para avaliação dos resultados: UCLA, Carter-Rowe e Constant-Murley. Na avaliação clínica usando o escore de Carter-Rowe, obtivemos a pontuação mediana de 95, sendo 67 resultados excelentes, 14 bons, 2 regulares e 11 ruins. Na avaliação do escore da UCLA, obtivemos 83 pacientes com resultados bons/excelen- tes e 11 pacientes com resultados ruins/razoáveis. Já com relação ao escore de Constant-Murley, obtivemos 65 pacientes com resultados excelentes, 18 bons, 10 razoáveis e 1 resultado ruim. Nos três escores, observamos que todos os pacientes com resultados razoáveis/ruins eram os que apresentavam recidiva das luxações.

Os resultados apresentados por Boileau et $\mathrm{al}^{28}$ em 91 pacientes evidenciaram pontuação média segundo os critérios de Carter-Rowe de 77,8 pontos (variando de 15 a 100 pontos). Balg e Boileau ${ }^{13}$ encontraram média de 81,5 pontos (variação de 10 a 100 pontos) no escore de Carter-Rowe após avaliação de 131 pacientes operados. Neri et al, ${ }^{29}$ analisando 11 pacientes, encontraram índice de Rowe de 74,5 pontos (variação de 35 a 100 pontos), e no escore da UCLA, média de 29,6 pontos (variando de 24 a 35 pontos), com resultados bons/excelentes em 8 pacientes (72,7\%), e razoáveis/ruins em 3 (27,3\%). Em trabalho avaliando 314 ombros de 302 pacientes, Godinho et al $^{30}$ obtiveram, no escore de Carter-Rowe, variação de 25 a 100 pontos, com média de 91,8 , e, no da UCLA, 33,8 pontos, com $97,6 \%$ de resultados bons/excelentes. Pudemos observar, em nossa casuística, uma correlação direta significativa entre o escore da UCLA e a idade $\left(r_{s}\right.$ $=0,250 ; p=0,015$ ). Isto significa que, quanto maior a idade, maior a pontuação no escore da UCLA.

Independente do método utilizado, a principal complicação pós-operatória é a recorrência das luxações, sendo estas associadas a fatores de risco previamente citados. Com isso, o uso de escores como o Instability and Severity Index Score (ISIS) pode indicar a técnica que promoverá maiores benefícios para os pacientes. O escore utiliza como critérios a idade no momento da cirurgia, o grau de participação esportiva, o tipo de esporte praticado, a frouxidão ligamentar, a presença de lesão de Hill-Sachs e a perda de contorno da glenoide, gerando 10 pontos no total. De acordo com os resultados de Balg e Boileau, ${ }^{13}$ pacientes com até seis pontos se beneficiam mais da técnica artroscópica, enquanto aqueles com menor pontuação são indicados para cirurgias abertas, principalmente de Latarjet.

As principais limitações do presente estudo incluem a sua natureza retrospectiva, que não permitiu a obtenção rigorosa 
de uma avaliação funcional no pré-operatório, o curto tempo de seguimento, e a distribuição não normal dos dados, que obrigou ao uso de testes não paramétricos.

\section{Conclusões}

Concluímos que o reparo artroscópico da instabilidade anterior do ombro apresenta resultados satisfatórios nos escores funcionais em curto e médio prazos, com baixo índice de complicações graves que gerem limitações, podendo ser um dos métodos de escolha para a maioria dos pacientes. Os resultados ruins/regulares nos escores estiveram associados às recidivas da luxação. A dor pós-operatória esteve associada à idade mais jovem no momento da cirurgia, e a limitação da rotação lateral está relacionada com o tipo de instabilidade (traumática).

\section{Conflito de Interesses}

Os autores declaram não haver conflito de interesses.

\section{Referências}

1 Veeger DH. "What if": the use of biomechanical models for understanding and treating upper extremity musculoskeletal disorders. Man Ther 2011;16(01):48-50

2 Perthes G. Uber Operationen bei tabitueller Schulterluxation. Dtsch Z Chir 1906;56:149-151

3 Bankart AS. Recurrent or habitual dislocation on the shoulder joint. BMJ 1923;2(3285):1132-1133

4 Bankart AS. The pathology and treatment of recurrent dislocation of the shoulder joint. Br J Surg 1938;26:23-29

5 Neviaser RJ, Benke MT, Neviaser AS. Mid-term to long-term outcome of the open Bankart repair for recurrent traumatic anterior dislocation of the shoulder. J Shoulder Elbow Surg 2017;26(11):1943-1947

6 Ahmed I, Ashton F, Robinson CM. Arthroscopic Bankart repair and capsular shift for recurrent anterior shoulder instability: functional outcomes and identification of risk factors for recurrence. J Bone Joint Surg Am 2012;94(14):1308-1315

7 Murray IR, Ahmed I, White NJ, Robinson CM. Traumatic anterior shoulder instability in the athlete. Scand J Med Sci Sports 2013;23 (04):387-405

8 Pulavarti RS, Symes TH, Rangan A. Surgical interventions for anterior shoulder instability in adults. Cochrane Database Syst Rev 2009;(04):CD005077

9 Rosa JR, Checchia CS, Miyazaki AN. Instabilidade anterior traumática do ombro. Rev Bras Ortop 2017;52(05):513-520

10 Randelli P, Ragone V, Carminati S, Cabitza P. Risk factors for recurrence after Bankart repair a systematic review. Knee Surg Sports Traumatol Arthrosc 2012;20(11):2129-2138

11 Grumet RC, Bach BR Jr, Provencher MT. Arthroscopic stabilization for first-time versus recurrent shoulder instability. Arthroscopy 2010;26(02):239-248

12 Virk MS, Manzo RL, Cote M, et al. Comparison of Time to Recurrence of Instability After Open and Arthroscopic Bankart Repair Techniques. Orthop J Sports Med 2016;4(06):2325967116654114
13 Balg F, Boileau P. The instability severity index score. A simple preoperative score to select patients for arthroscopic or open shoulder stabilisation. J Bone Joint Surg Br 2007;89(11):1470-1477

14 Castagna A, Markopoulos N, Conti M, Delle Rose G, Papadakou E, Garofalo R. Arthroscopic bankart suture-anchor repair: radiological and clinical outcome at minimum 10 years of follow-up. Am J Sports Med 2010;38(10):2012-2016

15 Petrera M, Patella V, Patella S, Theodoropoulos J. A meta-analysis of open versus arthroscopic Bankart repair using suture anchors. Knee Surg Sports Traumatol Arthrosc 2010;18(12):1742-1747

16 Bottoni CR, Smith EL, Berkowitz MJ, Towle RB, Moore JH. Arthroscopic versus open shoulder stabilization for recurrent anterior instability: a prospective randomized clinical trial. Am J Sports Med 2006;34(11):1730-1737

17 Ferreira Neto FAA, Camanho GL, Felix AM, et al. Tratamento artroscópico da instabilidade anterior do ombro: estudo retrospectivo de 159 casos. Acta Ortop Bras 2011;19(01):41-44

$18 \mathrm{Kim} \mathrm{SH}$, Ha KI, Cho YB, Ryu BD, Oh I. Arthroscopic anterior stabilization of the shoulder: two to six-year follow-up. J Bone Joint Surg Am 2003;85(08):1511-1518

19 Hayashida K, Yoneda M, Nakagawa S, Okamura K, Fukushima S. Arthroscopic Bankart suture repair for traumatic anterior shoulder instability: analysis of the causes of a recurrence. Arthroscopy 1998;14(03):295-301

20 Almeida Filho IA, Veado MA, Fim M, Corrêa LV, Júnior AE. Avaliação funcional do reparo artroscópicoda instabilidade anterior recidivante do ombro. Rev Bras Ortop 2012;47(02):214-221

21 Larrain MV, Montenegro HJ, Mauas DM, Collazo CC, Pavón F. Arthroscopic management of traumatic anterior shoulder instability in collision athletes: analysis of 204 cases with a 4- to 9-year follow-up and results with the suture anchor technique. Arthroscopy 2006;22(12):1283-1289

22 Enad JG, Gaines RJ, White SM, Kurtz CA. Arthroscopic SLAP repair in military patients. J Shoulder Elbow Surg 2007;16(03):300-305

23 Buscayret F, Edwards TB, Szabo I, Adeleine P, Coudane H, Walch G. Glenohumeral arthrosis in anterior instability before and after surgical treatment: incidence and contributing factors. Am J Sports Med 2004;32(05):1165-1172

24 Porcellini G, Campi F, Pegreffi F, Castagna A, Paladini P. Predisposing factors for recurrent shoulder dislocation after arthroscopic treatment. J Bone Joint Surg Am 2009;91(11):2537-2542

25 Flinkkilä T, Hyvönen P, Ohtonen P, Leppilahti J. Arthroscopic Bankart repair: results and risk factors of recurrence of instability. Knee Surg Sports Traumatol Arthrosc 2010;18(12):1752-1758

26 Voos JE, Livermore RW, Feeley BT, et al; HSS Sports Medicine Service. Prospective evaluation of arthroscopic bankart repairs for anterior instability. Am J Sports Med 2010;38(02):302-307

27 Calvo E, Granizo JJ, Fernández-Yruegas D. Criteria for arthroscopic treatment of anterior instability of the shoulder: a prospective study. J Bone Joint Surg Br 2005;87(05):677-683

28 Boileau P, Villalba M, Héry JY, Balg F, Ahrens P, Neyton L. Risk factors for recurrence of shoulder instability after arthroscopic Bankart repair. J Bone Joint Surg Am 2006;88(08):1755-1763

29 Neri BR, Tuckman DV, Bravman JT, Yim D, Sahajpal DT, Rokito AS. Arthroscopic revision of Bankart repair. J Shoulder Elbow Surg 2007;16(04):419-424

30 Godinho GG, França FO, Freitas JM, et al. Tratamento artroscópico da instabilidade anterior traumática do ombro: resultados a longo prazo e fatores de risco. Rev Bras Ortop 2008;43(05):157-166 